\title{
Análisis del uso de un simulador de colisiones para resolver un accidente de tráfico
}

\section{Analysis of the use of a collision simulator to solve a traffic accident}

\author{
Víctor López-Simó \\ Departament de Didàctica de la Matemàtica i les Ciències Experimentals (UAB) \\ David Ferrer Sànchez \\ INS Pius Font $i$ Quer
}

RESUMEN - Analizamos el uso que dan estudiantes de 2. ${ }^{\circ}$ de ESO a una simulación virtual sobre colisiones en un proyecto sobre física aplicada a accidentes de tráfico, donde los estudiantes actúan como peritos, abogados y jueces. Los resultados muestran que la simulación es el recurso más usado por los estudiantes en sus argumentaciones, en comparación con otros recursos como experimentos reales con coches de juguete y otros análisis cinemáticos como gráficas y cálculos. A su vez, observamos que estas argumentaciones a menudo son incompletas e incorporan concepciones erróneas sobre la naturaleza virtual e idealizada de la simulación. Identificamos, por tanto, la necesidad de abordar no solo la comprensión conceptual y procedimental de las simulaciones, sino también epistémicas, durante su proceso de enseńanza y aprendizaje.

PALABRAS CLAVE: Simulación; Modelo; Colisión; Física; Argumentación.

ABSTRACT - We analyse how secondary students use a collision simulator within an applied physics project about traffic accidents, in which students act as experts, lawyers and judges. The results show that this simulator is the most used resource by students in their arguments, compared to other resources such as real experiments with toy cars and other kinematic analyses such as graphs or calculations. We also note that these arguments are often incomplete, and they include misconceptions about the virtual and idealized nature of simulations. We identify, therefore, the need to address not only the conceptual and procedural understanding of simulations, but also its epistemology.

KEYWORDS: Simulation; Model; Collision; Physics; Argumentation. 


\section{INTRODUCCIÓN}

En los últimos años la escuela está viviendo la eclosión de enfoques didácticos que abogan por un papel más activo del alumnado en su proceso de aprendizaje y de unas prácticas educativas más significativas y vivenciales. La progresiva implantación del trabajo por proyectos (ABP) en muchos centros de nuestra geografía es una muestra de este momento de efervescencia (Márquez y Sanmartí, 2017), aunque hay que tener en cuenta que estas propuestas metodológicas no son una novedad en sí mismas, pues tienen su origen en las propuestas pedagógicas de Dewey o Kilpatrick en las primeras décadas del siglo XX (Hasni et al., 2016). En el ámbito STEM, se han identificado algunas tensiones que surgen a la hora de diseñar proyectos $\mathrm{ABP}$, como la selección de qué reto se propone al alumnado, qué nivel de interacción entre diferentes disciplinas, qué grado de relevancia se da al fenómeno o contexto de estudio, o qué forma se da a la evaluación de aprendizaje del alumnado (Pérez et al., 2020).

El proyecto CRASH (Domènech-Casal et al., 2018) es un ejemplo de esta oleada de proyectos escolares, diseñado a través de la colaboración entre profesorado y llevado a la práctica en más de una veintena de centros de secundaria en los cuatro últimos cursos. En este proyecto se propone a los estudiantes la resolución de un caso ficticio, pero con una gran dosis de realismo: un accidente de tráfico con varios vehículos implicados, marcas en la carretera y declaraciones de testigos. A lo largo del proyecto el alumnado debe recopilar diferentes informaciones, simular cómo ha sido el accidente (mediante cálculos, estimaciones y experimentos reales y virtuales) y resolver el caso a través de una teatralización donde los propios estudiantes simulan ser las partes implicadas: abogados, peritos judiciales e incluso jueces. Esta teatralización en el marco de una narración ficticia es lo que se denomina juego de rol (Matas, 2003), una estrategia didáctica con múltiples potencialidades para el desarrollo de habilidades y actitudes en el ámbito científico (Blanco-López et al., 2017). Además, de este modo, el proyecto no solo promueve habilidades científicas, sino que incide en la educación vial del alumnado (Montané et al., 2007).

\section{MARCO TEÓRICO}

\section{El desarrollo de prácticas científicas en el aula a través del estudio de casos, juegos de rol y argumentación}

En esta eclosión de una enseñanza-aprendizaje significativa, enfocada hacia el desarrollo de competencias y con un papel más activo del estudiante, el marco de las prácticas científicas está generando una gran adhesión en el área de la didáctica de las ciencias. Este marco sugiere que la educación científica debe ofrecer al alumnado la experiencia de participar de unas prácticas científicas auténticas, genuinas y análogas a las de la ciencia real (Osborne, 2014). Esta manera de entender las clases de ciencia se refleja en el nuevo currículum de los Estados Unidos de América, donde la National Research Council concreta ocho prácticas científicas que promover en el aula: plantearse preguntas sobre fenómenos naturales relevantes para la ciencia; desarrollar y usar modelos científicos; planificar y llevar a cabo investigaciones; analizar e interpretar datos experimentales; usar pensamiento computacional y matemático; construir explicaciones y diseñar soluciones; argumentar científicamente basándose en pruebas; y comunicar a la comunidad los resultados de la actividad científica. Además, se trata de una propuesta coherente con el marco de las competencias científicas (Crujeiras y Jiménez-Aleixandre, 2012).

Así, las clases de ciencias no deben centrarse en la asimilación de contenidos que transmite el docente (las ideas o modelos científicos descritos en el libro de texto tradicional), sino en el proceso de construcción y refinamiento de las explicaciones o modelos que construyen progresivamente los estudiantes sobre los fenómenos del mundo natural a los que se enfrentan. Coincidiendo con la idea 
de Izquierdo et al. (1999) según la cual el auténtico aprendizaje de la ciencia escolar es, en el fondo, el proceso de "pensar, hacer y hablar» ciencia, Osborne (2014) propone considerar tres esferas interrelacionadas de la práctica científica en el aula: modelización, indagación y argumentación (Couso et al., 2020). Así, la modelización puede entenderse como el proceso que implica desarrollar, evaluar y refinar explicaciones científicas sobre fenómenos naturales (Justi, 2006; Gutiérrez, 2004) ; la indagación es el proceso que conlleva diseńar y llevar a cabo experimentos y analizar e interpretar datos (Minner et al., 2010); y la argumentación puede entenderse como comunicar las ideas propias y las interpretaciones de los resultados para persuadir a los demás de su validez (Erduran y Jiménez-Aleixandre, 2007). Respecto a esta dimensión argumentativa, autores como Jiménez-Aleixandre et al. (2003) han analizado la calidad de las operaciones argumentativas del alumnado de afirmación y justificación, así como las operaciones epistémicas de construcción de explicaciones a través de datos.

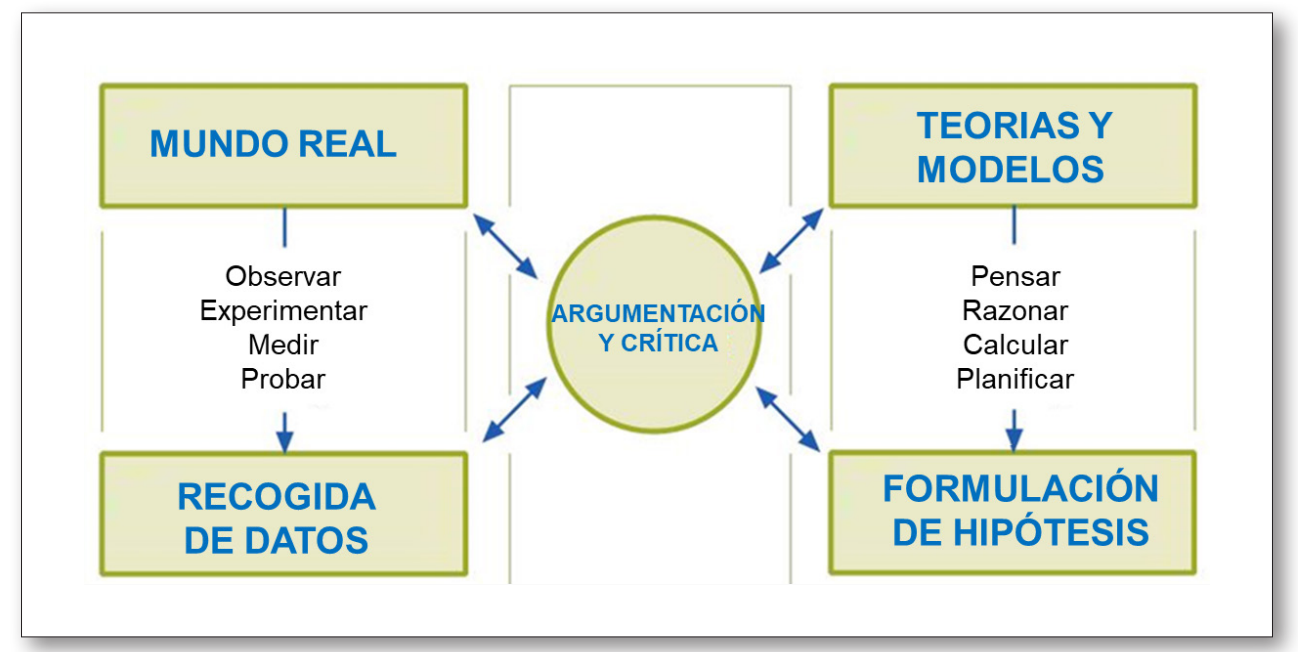

Fig. 1. Modelo de la actividad científica propuesto por Osborne (2014).

Las estrategias y enfoques para involucrar al alumnado en estas prácticas científicas son múltiples, híbridas y complementarias, y su caracterización exhaustiva escapa al alcance de este documento, pues va desde la ciencia presentada a través de contexto, la modelización basada en la indagación, las controversias sociocientíficas, el aprendizaje basado en proyectos, el juego de rol, etc. Dentro del ABP, Domenech-Casal (2019a), inspirado en prácticas educativas propias de otros ámbitos como la genética forense, propone el enfoque "Resolución de un caso», que incluye aspectos como una situación inicial contextualizada (real o verosímil), que a su vez contiene un problema para resolver a partir de la interpretación y ponderación de pruebas, el uso instrumental de modelos teóricos y dinámicas de discusión y argumentación. Según este autor, el método de caso dirigido es un tipo concreto de estudios de caso: se proporcionan al alumnado nuevas pruebas de forma paulatina (Glew, 2003; Herreid, 2003), se emula la forma en que en la ciencia aparecen las evidencias y esto provoca cambios en las conclusiones. En paralelo a la resolución del problema, los alumnos reciben clases teóricas sobre los modelos científicos implicados para que establezcan conexiones entre dichos modelos científicos y el contexto en que se ubica el problema que debe resolverse.

La resolución de un caso puede hacerse también a través de un juego de rol, es decir, una dinámica donde los estudiantes simulan ser un personaje ficticio dentro de una narrativa. El juego de rol ha sido una estrategia didáctica señalada en la literatura del ámbito de la enseñanza de las ciencias con múltiples potencialidades para el desarrollo de habilidades y actitudes. Blanco-López et al. (2017) señalan el juego de rol como una de las estrategias eficaces para promover el pensamiento crítico en el 
ámbito científico, y Anta et al. (2019) añaden que también tiene potencial en la formación inicial de profesorado. González-Sánchez (2017) identifica una percepción positiva del alumnado de ESO de esta estrategia didáctica para mejorar las habilidades de argumentación y de hablar en público, para aprender a defender argumentos y discriminar información fiable; y Pons y de Soto (2020) identifican un impacto positivo en la motivación hacia las ciencias en bachillerato.

\section{El papel de las simulaciones virtuales para el desarrollo de prácticas científicas en el aula}

En consonancia con este planteamiento, que aboga por involucrar a los estudiantes en "hacer ciencia», el desarrollo de la tecnología digital ha permitido y facilitado el crecimiento de las simulaciones y laboratorios virtuales con finalidades educativas. Estos recursos se pueden definir como representaciones dinámicas generadas por computadora de modelos, procesos o fenómenos del mundo real (Smetana y Bell, 2012). Pueden identificarse como mediadores en la relación entre la realidad y los modelos o teorías científicas, y ofrecen posibles interacciones entre los modelos mentales de los estudiantes sobre un tema determinado y los modelos científicos subyacentes tras la simulación (Evagorou et al., 2009; Gutiérrez y Pintó, 2004). Se han hallado resultados sobre su eficiencia para representar fenómenos científicos complejos sin ningún tipo de riesgo experimental (Smetana y Bell, 2012), para ayudar al alumnado a comprender de conceptos científicos difíciles (Plass et al., 2012) y para fomentar la interactividad y el dinamismo (Ryoo y Linn, 2012), así como el compromiso y la motivación del alumnado (Honey y Hilton, 2011). Por otro lado, algunos riesgos de utilizar la simulación se han destacado en la literatura, como la falta de realismo en comparación con los escenarios reales basados en problemas (Byrne et al., 2010), dificultades para comprender las representaciones virtuales (López y Pintó, 2017) o puntos de vista parciales sobre los fenómenos naturales, causados por los resultados preprogramados excesivamente idealizados que muestran estas herramientas educativas (Vallverdú, 2014). En este sentido, Pessanha (2014) relacionó los obstáculos epistemológicos definidos por Bachelard en el uso de simulaciones de física en secundaria.

Otra cuestión particular que ha centrado parte del debate sobre el uso de estos recursos en el aula de ciencia, y que toma una gran relevancia en nuestro caso, es hasta qué punto las simulaciones y laboratorios virtuales pueden complementar o incluso sustituir los experimentos físicos realizados en laboratorios reales. La literatura no aporta una respuesta clara a esta cuestión. Klahr et al. (2006) compararon el efecto que tiene el soporte real (interactuar con objetos físicos) y virtual (interactuar mediante el ordenador) en el aprendizaje de estudiantes de escuela media que experimentaban con coches de juguete, pero no encontraron diferencias significativas. En investigaciones parecidas en el contexto de enseńanza de la electricidad (Zacharia, 2007), termodinámica (Zacharia et al., 2008) u óptica (Olympiou y Zacharia, 2012), se observó que la combinación de simulaciones y experimentos manipulativos mejoró la comprensión conceptual de los estudiantes más que el uso exclusivo del soporte manipulativo. Jong et al. (2013) afirman que los experimentos virtuales aportan un valor añadido para los experimentos reales cuando muestran a los estudiantes fenómenos no observables, se señala la información relevante y adaptada al estudiante y permite realizar múltiples experimentos en una pequeña cantidad de tiempo. Finalmente, para el caso concreto de la educación vial, Fernández, Ávila y Milanés (2017) han observado un impacto positivo de las animaciones 3D en el aprendizaje de la educación vial en educación secundaria.

\section{La simulación Collision Lab}

Una de las muchas simulaciones virtuales que existen disponibles para la enseñanza y aprendizaje de las ciencias es la denominada Collision Lab (PhET, 2020). Se trata de una simulación computacional 
elaborada por PhET de Colorado (Wieman et al., 2008) para reproducir colisiones entre dos cuerpos circulares. A partir de los parámetros iniciales de posición y velocidad definidos por el usuario, y en función de si se quiere reproducir un choque elástico o inelástico, en la simulación aparece representado el choque, que funciona a partir de las leyes dinámicas de conservación del momento lineal y, en caso de los choques elásticos, de la energía cinética del sistema. Así, cambiando los parámetros iniciales del choque, se puede reproducir tantos choques como desee el usuario. Por ejemplo, en la figura 2 se observan vectores que representan la dirección e intensidad de la velocidad en cada momento, y cómo después de la colisión el cambio de velocidad es mayor en el objeto 1, ya que su masa es mucho menor.

Hay que tener en cuenta que el conocimiento en profundidad y matematizado de estas leyes de conservación no son un objetivo de aprendizaje de la ESO (López et al., 2020), sino que se estudian en Física de Bachillerato, pero sí que es posible deducir algunas relaciones cualitativas en edades más tempranas, como por ejemplo la influencia de la masa y la velocidad de cada cuerpo circular en la colisión. Además, como ocurre con muchas simulaciones, ofrece un alto grado de interactividad inmediata que promueve y facilita su uso por parte del alumnado (Ryoo y Linn, 2012).

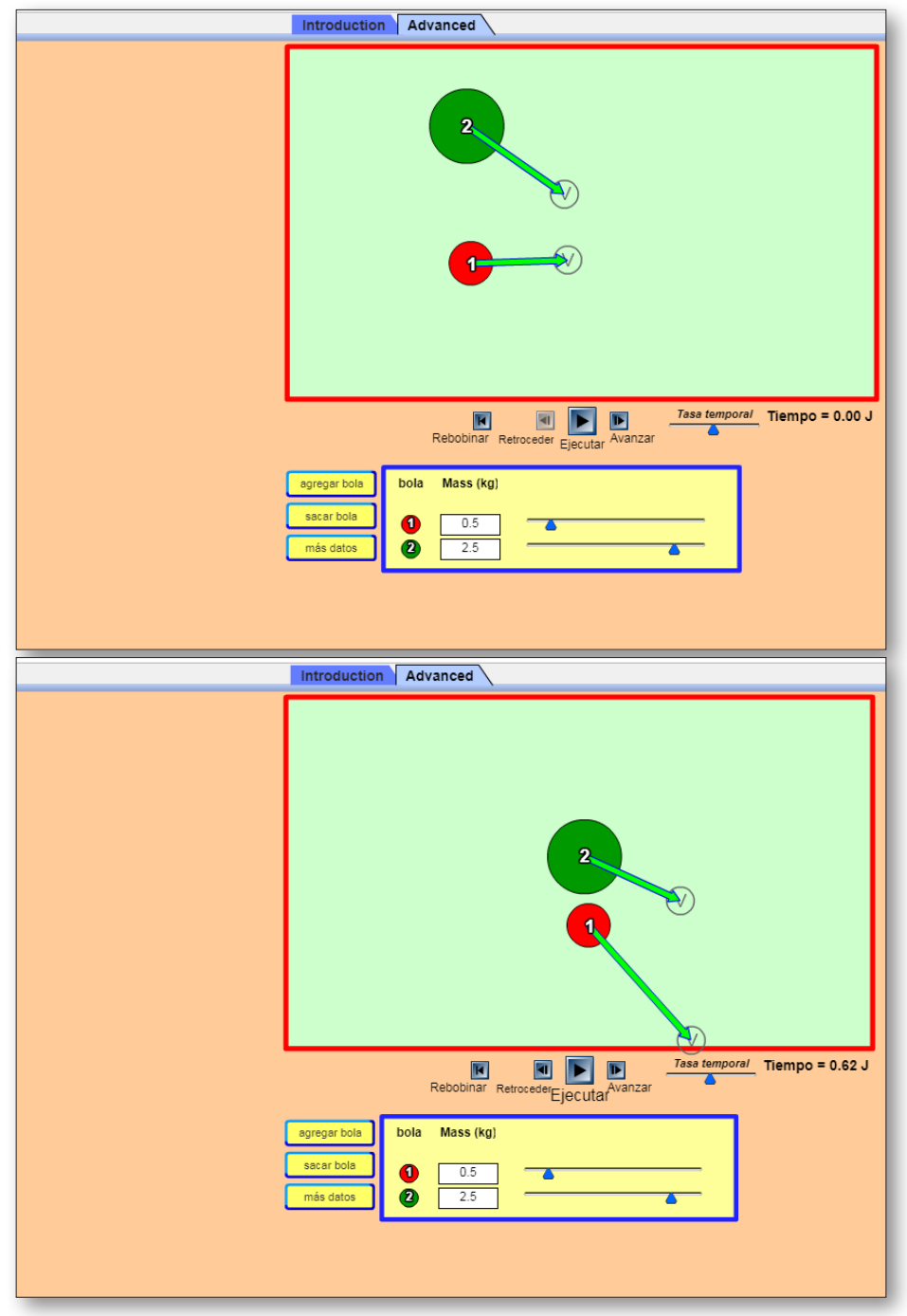

Fig. 2. Situación antes y después de la colisión de dos objetos circulares de masa distinta $(0,5$ y $2,5 \mathrm{~kg})$. 


\section{OBJETIVOS Y PREGUNTAS DE INVESTIGACIÓN}

En esta investigación nos proponemos conocer cómo es el uso que hacen de la simulación Collision Lab los estudiantes de 2..$^{\circ}$ de ESO de dos centros públicos en Cataluña, en el marco de un juego de rol sobre resolución de un accidente de tráfico.

Para ello, planteamos dos preguntas de investigación:

- Pregunta de investigación 1: ¿Cómo de frecuente es Collision Lab como prueba para argumentar sobre un accidente de tráfico en comparación con otros tipos de prueba?

- Pregunta de investigación 2: ¿Cómo argumentan los estudiantes cuando usan la simulación Collision Lab para para apoyar su versión del accidente de tráfico?

\section{METODOLOGÍA}

\section{Contexto y participantes}

Durante el curso 2018-19 dos profesores de ciencias, autores de la publicación, implementamos el proyecto CRASH en la asignatura de Física y Química en dos centros públicos de secundaria en Cataluña: el INS Pau Vila, situado en la ciudad de Sabadell, y el INS Pius Font i Quer, situado en la ciudad de Manresa. En ambos casos involucramos a dos grupos clase de $2 .^{\circ}$ de ESO, todos ellos de entre 30 y 31 estudiantes. Los 4 grupos clase (2 en cada centro) eran grupos heterogéneos de alumnado, con un $50 \%$ de chicos y un $50 \%$ de chicas, y con un posicionamiento hacia el aprendizaje de las ciencias y un rendimiento académico muy variados. Ambos centros reciben alumnado de los barrios colindantes, con un el perfil socioeconómico medio y medio-alto.

En este proyecto se propone a los estudiantes la resolución de un caso ficticio, pero con una gran dosis de realismo: un accidente de tráfico con varios conductores implicados, marcas en la carretera y declaraciones de testigos (figura 3). Poniéndose en el papel de peritos judiciales, de abogados y de jueces, los estudiantes deben aprender conceptos básicos de física y, a la par, construir una reproducción lo más verosímil posible de qué responsabilidad tenían los conductores implicados (Domènech-Casal et al., 2018). 


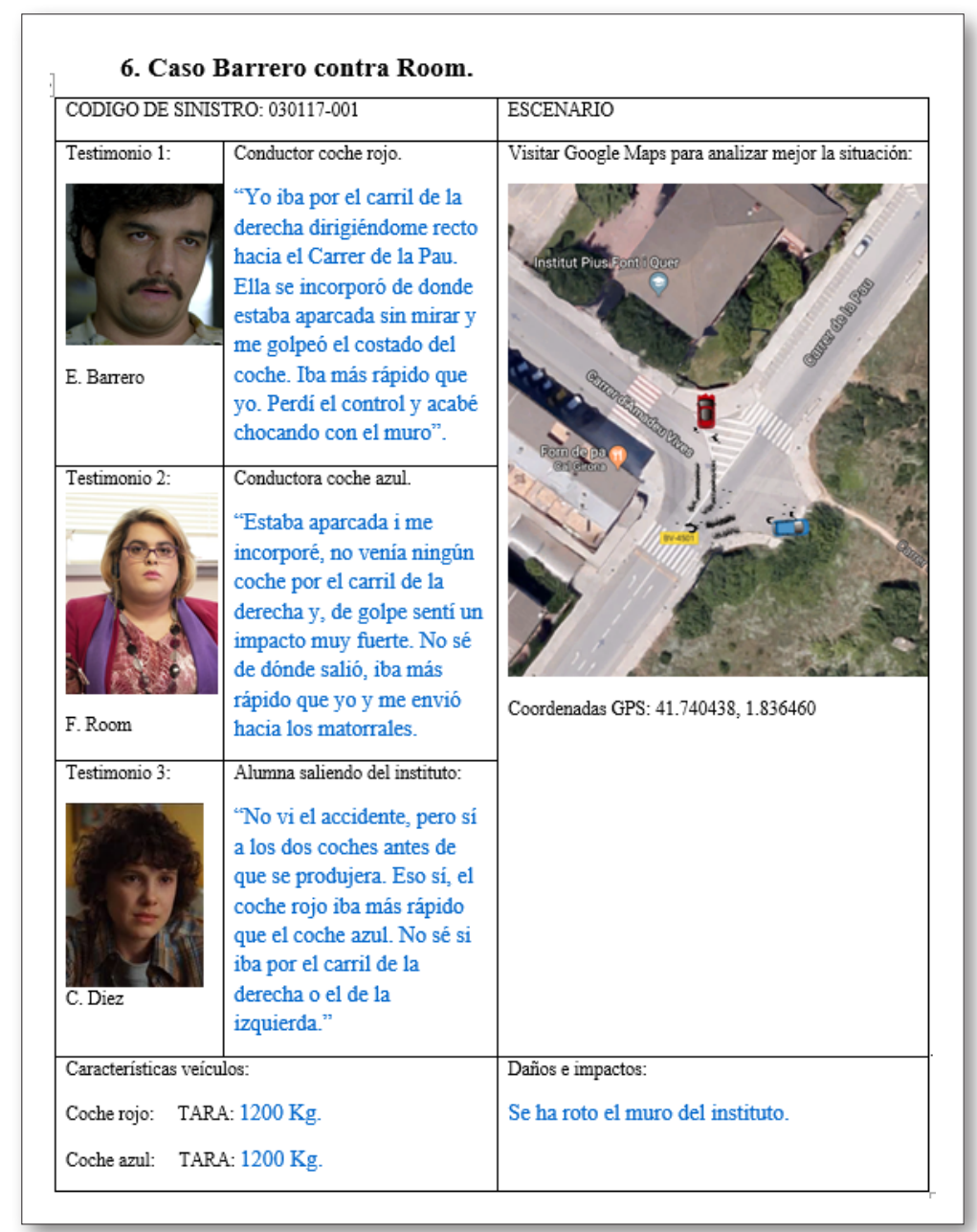

Fig. 3. Ficha de uno de los casos con datos relevantes del caso.

El desarrollo del proyecto incluye diferentes actividades (figura 4), en las que los estudiantes deben aprender, de forma progresiva, a representar e interpretar gráficos posición-tiempo, a distinguir entre movimientos uniformes y no uniformes, o a explicar la relación entre masa y cambio de velocidad (2. ${ }^{\text {a }}$ ley de Newton), entre otros.

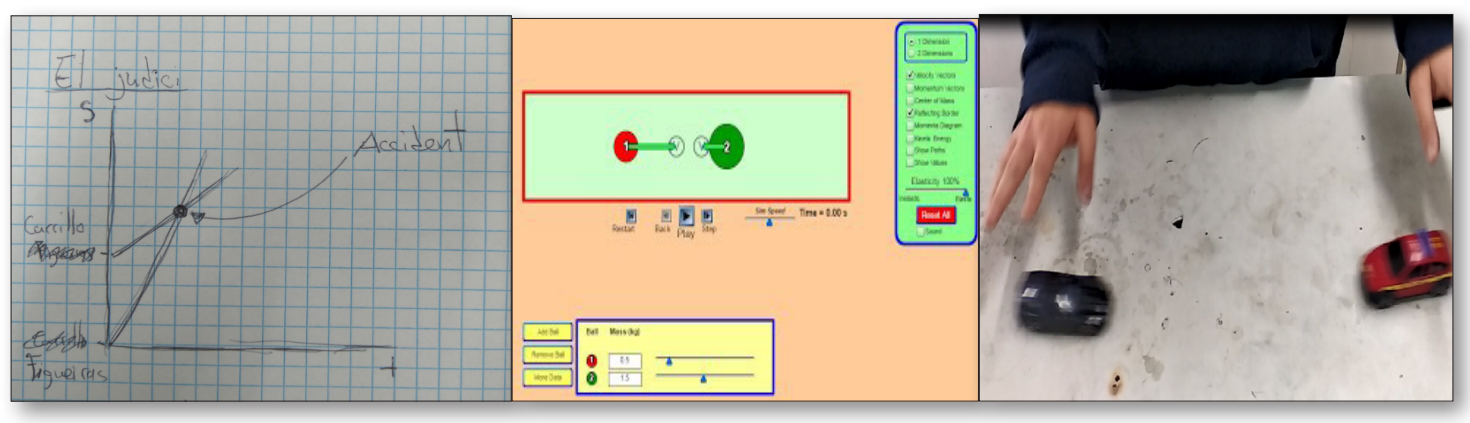

Fig. 4. Tres ejemplos de actividades en las que se involucra el alumnado a lo largo del proyecto: elaboración de gráficas posición tiempo, simulación con Collision Lab y simulación con coches de juguete. 
El proyecto CRASH termina con una resolución de un caso como el de la figura 3, en el que los estudiantes, en pequeños grupos (de 3 o 4 estudiantes), debían aportar una versión narrada de cómo ha sido alguno de los accidentes. Para ello, tenían que exponer oralmente delante del resto de la clase su versión de los hechos, durante un periodo corto de tiempo (unos 10 minutos). La narrativa para presentar esta versión del accidente difirió ligeramente en ambos centros. En el caso del INS Pau Vila, los estudiantes simulaban ser abogados de acusación, abogados de defensa y jueces, y celebraron un juicio oral en la que los jueces debían dirimir sobre la culpabilidad de los responsables implicados en el accidente de tráfico. El resto de los estudiantes del grupo clase hacían de público del juicio (ver figura 5). En cambio, en el INS Pius Font i Quer los estudiantes simulaban ser peritos judiciales que mostraban sus análisis y su versión de los hechos al juez (el profesor).

A pesar de esta diferencia en la forma de presentar las evidencias, en ambos casos los estudiantes debían ofrecer la versión más verosímil y consistente de cómo había sucedido el accidente del caso que se les había adjudicado, y para ello, durante su exposición oral, los estudiantes podían incluir en su elección cualquier tipo de prueba pericial para reforzar su versión de los hechos, a partir de las diferentes actividades realizadas a lo largo del proyecto: vídeos de experimentos con coches de juguetes, vídeos de experimentos con la simulación Collision Lab, cálculos y gráficos realizados por los propios estudiantes, etc. En ambos casos los jueces (en un caso los propios estudiantes y en el otro el profesor) eran los responsables de hacer preguntas para poner a prueba la solidez de los argumentos usados por las partes y los peritos.

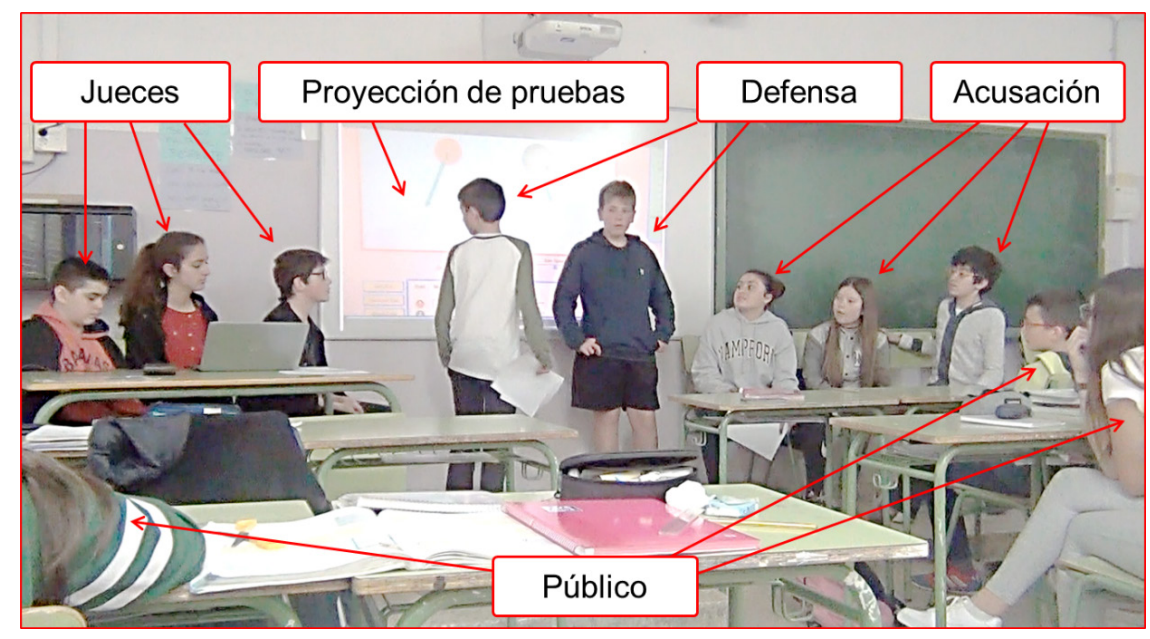

Fig. 5. Representación teatralizada de un juicio oral con jueces, defensa, acusación y público, celebrada en el INS Pau Vila. Todos los menores cuentan con un permiso para publicar sus imágenes.

El desarrollo de los argumentos presentados oralmente para exponer su versión de los hechos llevó a los autores del artículo a interesarnos por cuál era el uso que los estudiantes hacían de la simulación Collision Lab, cuyo uso, como ya hemos dicho, era opcional, pero resultó ser muy versátil. Por este motivo decidimos analizar cuál había sido su uso, a través de la investigación que aquí presentamos.

\section{Recogida de datos}

Ambos profesores recogimos datos durante las sesiones, en las que los estudiantes presentaban oralmente y en pequeños grupos los resultados de su estudio de caso. En total se registraron, a través de ví- 
deo y de notas de campo, 8 presentaciones en cada uno de los dos institutos, de manera que se disponía de un total de 16 unidades de análisis. Esta recogida de datos se realizó entre febrero y abril de 2019.

\section{Análisis de qué tipos de recursos usan en la resolución del caso}

Así, para responder a la primera pregunta de investigación, cada profesor elaboró una tabla donde se recogieron las diferentes notas de campo que habíamos escrito durante la exposición de la resolución del caso en cada grupo. Posteriormente, cada tabla se complementó con nuevas notas obtenidas a partir del visionado posterior de los vídeos. Una vez hecho esto, ambos profesores pusimos en común ambas tablas, y acordamos generar un sistema de categorías para clasificar los recursos que habían usado los estudiantes que permitiera posteriormente hacer un recuento de la prevalencia de uso de estos recursos, para así poder compararlos entre ellos y responder a la pregunta de investigación 1.

De este modo, para establecer este sistema de categorías, en primer lugar, se descartaron todas las pruebas de carácter anecdótico o fantasioso, que no tenían relación con el contenido de física estudiado en clase. Así, se dejaron de lado las imágenes de cámaras de seguridad o los testigos inventados por los estudiantes, registros de llamadas de teléfono móvil, que demostraban que los conductores lo estaban usando en el momento del accidente, o normas de circulación inventadas. Una vez que nos quedamos solamente con las pruebas relacionadas con ideas de cinemática y dinámica (posición, velocidad, fuerzas, masa, trayectoria, impulso, etc.), agrupamos de forma inductiva las pruebas utilizadas en cada presentación, con lo que se obtuvo un sistema con 4 tipologías:

- Los experimentos reales realizados con coches de juguete. En este tipo de pruebas, los estudiantes lanzaban coches sobre una mesa, teniendo en cuenta la posición y la velocidad antes del choque, para determinar así la posición y velocidad después de la colisión. En algunos casos, los estudiantes ajustaban la masa de los vehículos añadiendo trozos de plastilina a los coches.

- Los experimentos virtuales realizados con Collision Lab. Igual que en las pruebas anteriores, los estudiantes determinaban la masa, la posición y la velocidad de cada vehículo antes del choque, y la propia simulación determinaba la posición y velocidad después de este.

- Las animaciones manuales realizadas con coches de juguete, con otros objetos físicos o con objetos virtuales con presentaciones PPT. A diferencia de los casos anteriores, no existe experimento en sí mismo, sino que los estudiantes narran mediante una animación con la que ellos deciden manualmente el comportamiento físico de cada vehículo durante la colisión.

- Los gráficos cinemáticos de posición-tiempo o velocidad-tiempo, así como cálculos usando las ecuaciones del MRU. Son reconstrucciones realizadas por los estudiantes sin un origen empírico o experimental, sino que son valores inventados por los estudiantes, a menudo para tratar de explicar lo que creen que ha pasado.

Estas 4 categorías, a su vez, pueden organizarse a través de 2 dimensiones, tal y como se muestra en la tabla 1. Por un lado, si las pruebas que presenta el alumnado operan con representaciones realistas (se asemejan visualmente a un vehículo) o abstractas (vectores y magnitudes cinemáticas). Por otro, si lleva asociada una práctica de exploración o de expresión. Así, mientras que en la columna de la izquierda el estudiante «explora» el posible fenómeno simulado (ya sea a través de una maqueta o una simulación computacional), en la de la derecha «expresa» su idea de cómo ha podido ser el accidente, pero no opera con datos que haya simulado. 
Tabla 1. Clasificación propuesta de recursos utilizados por el alumnado para la resolución del caso de accidente de tráfico, usada para el análisis de qué tipos de recursos usan en la resolución del caso.

\begin{tabular}{|l|c|c|}
\hline & $\begin{array}{l}\text { Exploración (opera con datos simula- } \\
\text { dos y los compara con los datos reales) }\end{array}$ & $\begin{array}{l}\text { Expresión (intenta dar sentido a los } \\
\text { datos reales sin usar datos simulados) }\end{array}$ \\
\hline $\begin{array}{l}\text { Se opera con representaciones rea- } \\
\text { listas de los choches y su compor- } \\
\text { tamiento }\end{array}$ & Experimento real & Animación manual \\
\hline $\begin{array}{l}\text { Se opera con representaciones abs- } \\
\text { tractas de magnitudes físicas (vecto- } \\
\text { res, magnitudes cinemáticas). }\end{array}$ & Experimento virtual &
\end{tabular}

\section{Análisis de la argumentación que hacen cuando usan la simulación Collision Lab}

A su vez, para responder a la segunda pregunta, se analizaron y compararon los distintos episodios en los que la discusión de los estudiantes giraba en torno al uso del Collision Lab. Al hacerlo, pudimos observar diferencias en la manera de relacionar los elementos y entidades del propio caso «real» (las marcas de la carretera o la posición final de los vehículos, como los presentados en la figura 1) y los elementos y entidades que aportaba la simulación (las trayectorias en un choque ideal, como en la figura 3), que son una representación externa del modelo subyacente tras la simulación (Evagorou et al., 2009). A partir de esta idea, e inspirándonos en el diagrama de Osborne (2014) presentado en la figura 1, propusimos concebir la argumentación del alumnado como la forma en la que estos relacionan las entidades del mundo real con las provenientes de teorías y modelos, tal y como expresamos en la figura 6 .

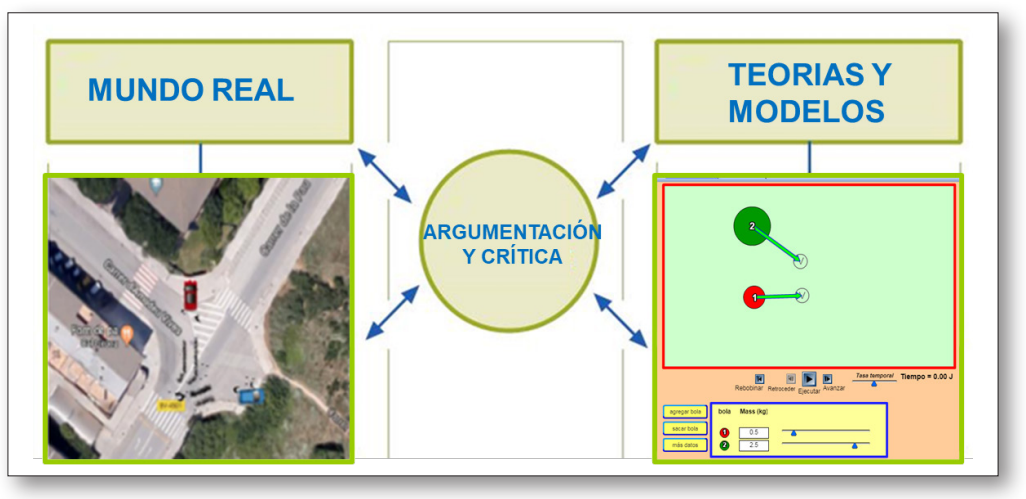

Fig. 6. Aplicación del diagrama de prácticas científicas (figura 1), donde los datos del caso (figura 3) se sitúan en «el mundo real», y la simulación del choque (figura 2) se sitúa en "teorías y modelos». 
A su vez, a la hora de analizar los argumentos construidos por los estudiantes, había que tener en cuenta si estaban hablando de lo que sucede antes del choque o después de este. Y es que las leyes físicas de una colisión que sirven para modelizar un accidente de tráfico como los planteados a los estudiantes son, en el fondo, la relación matematizada entre unos valores iniciales antes de la colisión (masa, velocidad, dirección, posición...) y unos valores finales resultantes de esta colisión (velocidad, dirección y posición después del choque). Así, si nos basamos en el diagrama de la figura 6 para clasificar qué elementos hablan los estudiantes, y añadimos la dimensión «antes/después de la colisión», podemos hablar de cuatro elementos de discusión, que hemos codificado como RA (real antes), RD (real después), VA (virtual antes) y VD (virtual después).

\section{Tabla 2 .}

Taxonomía de los elementos de discusión

sobre la relación entre simulación y fenómeno real, usada para

el análisis cualitativo del uso de la simulación en la resolución de los casos.

\begin{tabular}{|l|l|l|}
\hline & \multicolumn{1}{|c|}{ Mundo Real } & \multicolumn{1}{c|}{ Teorialmodelo Virtual } \\
\hline $\begin{array}{l}\text { Movimiento Antes } \\
\text { de la colisión }\end{array}$ & $\begin{array}{l}{[\mathrm{RA}] \text { Es desconocido por el estudiante, }} \\
\text { aunque puede estar guiado por los testigos }\end{array}$ & $\begin{array}{l}\text { [VA] Lo define el estudiante cuando intro- } \\
\text { duce los parámetros de la colisión }\end{array}$ \\
\hline $\begin{array}{l}\text { Movimiento Después } \\
\text { de la colisión }\end{array}$ & $\begin{array}{l}{[\mathrm{RD}] \text { Se deduce de la ficha del caso, aun- }} \\
\text { que con margen para la interpretación }\end{array}$ & $\begin{array}{l}\text { [VD] Lo genera la simulación a partir de los } \\
\text { parámetros antes de la colisión }\end{array}$ \\
\hline
\end{tabular}

Estos cuatro elementos serán los que servirán para definir las categorías del análisis cualitativo del uso de la simulación en la resolución de los casos.

\section{RESULTADOS Y DISCUSIÓN}

\section{Para la pregunta de investigación 1: ¿Cómo de frecuente es Collision Lab como prueba para argumentar sobre un accidente de tráfico en comparación con otros tipos de prueba?}

En la tabla 3 presentamos el número de estudiantes que, durante su exposición oral, eligieron usar cada tipo de pruebas, de acuerdo con la clasificación presentada anteriormente. Hay que tener en cuenta que la suma de los valores de las distintas filas de la tabla 3 no coincide con el número total de grupos (16 en cada centro), ya que algunos estudiantes presentaban más de una prueba y otros no presentaban ninguna prueba relevante desde el punto de vista físico.

Tabla 3.

Número de grupos de estudiantes que usan cada tipo de recurso.

\begin{tabular}{|l|c|c|c|}
\hline & $\begin{array}{c}\text { INS Pau Vila } \\
\text { N=16 grupos }\end{array}$ & $\begin{array}{c}\text { INS Pius Font i Quer } \\
\text { N=16 grupos }\end{array}$ & $\begin{array}{c}\text { Total } \\
\text { N }=32 \text { grupos }\end{array}$ \\
\hline Experimento real (con coches de juguete) & $5(31 \%)$ & $1(6 \%)$ & $6(19 \%)$ \\
\hline Experimento virtual (con Collision Lab) & $11(69 \%)$ & $8(50 \%)$ & $19(59 \%)$ \\
\hline $\begin{array}{l}\text { Animación manual (con coches de juguete o } \\
\text { imágenes dinámicas) }\end{array}$ & $2(13 \%)$ & $5(31 \%)$ & $7(22 \%)$ \\
\hline Gráficos y cálculos cinemáticos & $2(13 \%)$ & $1(6 \%)$ & $3(9 \%)$ \\
\hline
\end{tabular}


De acuerdo con los valores presentados en la tabla 3, se observa que las simulaciones con Collision Lab (clasificadas como experimento virtual en la tabla 1) son el tipo de pruebas más utilizadas en ambos centros, siendo usadas por el 69 y el 50 \%, respectivamente. En segundo lugar, se encuentran las simulaciones con coches de juguete en el caso del INS Pau Vila (31\%), mientras que en el INS Pius Font i Quer el segundo recurso más usado son las animaciones manuales $(31 \%)$. A pesar de estas diferencias entre ambos centros, al usar la prueba Chi, estas resultan no ser estadísticamente significativas $(\mathrm{p}=0,24)$, ya que se trata de una muestra muy pequeña.

Hay que tener en cuenta que en su momento no se preguntó explícitamente a los estudiantes el porqué de su elección, cosa que, visto en perspectiva, es una importante limitación, ya que no nos permite responder cuál fue el motivo de esta prevalencia de la simulación. No obstante, basándonos en la literatura, podemos identificar posibles causas, como la facilidad que ofrece Collision Lab para reproducir virtualmente el fenómeno en comparación con los coches de juguete reales (Klahr et al., 2006), o simplemente el hecho de que la simulación promueve una mayor motivación e interés en los estudiantes (Honey y Hilton, 2011). Otros posibles factores podrían ser la apariencia de rigor científico que ofrece la simulación frente a las demás representaciones, o incluso la predilección de los estudiantes por este tipo de representaciones debido a su cultura multimedia.

\section{Para la pregunta de investigación 2: ¿Cómo argumentan los estudiantes cuando usan la simulación Collision Lab para apoyar su versión del accidente de tráfico?}

Dentro de estos 19 grupos que optaron por usar la simulación como prueba pericial (un 59\% del total de grupos), se observaron diferencias en qué uso hicieron de esta, tanto si comparamos los grupos entre ellos como si comparamos los cambios en el discurso de un mismo grupo a lo largo de la presentación. Como se ha expuesto anteriormente, usar la simulación como prueba durante la reconstrucción de los hechos debía servir para discutir sobre la velocidad de los dos vehículos (usando expresiones como «los dos iban igual de rápido», «uno iba mucho más rápido que el otro», «el segundo vehículo estaba parado», etc.) y su posición relativa en el momento de la colisión («le golpeó por detrás», «le golpeó de lado", etc.). Para analizar esta cuestión, en la tabla 2 hemos propuesto una taxonomía de los elementos de discusión sobre la relación entre simulación y fenómeno real: RA (real antes), RD (real después), VA (virtual antes) y VD (virtual después).

Esta clasificación sobre los elementos de discusión permite analizar cómo los estudiantes construyen sus argumentos viendo la manera en la que conectan estos elementos entre ellos. Hemos definido tres niveles argumentativos, inspirándonos en el lenguaje de la lógica formal, que presentamos a continuación.

\section{Nivel de argumentación 1: Si VA $\rightarrow$ demuestro $R A$}

En sus presentaciones orales, algunos estudiantes usaban los valores de posición y velocidad que aparecían en la simulación antes del choque (VA), y que ellos mismos habían definido al usar la simulación, como si esto permitiera demostrar cuál había sido el movimiento real de alguno de los vehículos (RA). Es decir, que en su argumentación concebían la simulación como una evidencia en sí misma, sin necesidad de que tuviera relación con los datos a los que se enfrentaban.

En el siguiente ejemplo, se muestra un diálogo entre un chico que ejerce de acusación contra el conductor de un camión rojo (e intenta demostrar que este camión iba más rápido de la velocidad permitida) y una chica que ejerce de defensa del camión (e intenta convencer al juez que el camión conducía despacio): 
Defensa: Un testigo dijo que el automóvil conducía por encima de la velocidad permitida y que el camión conducía más despacio.

Acusación: No. Como se indica aquí [señalando el vector velocidad del objeto que simula ser el camión en la simulación que el mismo aporta como prueba], el camión iba a más de $20 \mathrm{~km} / \mathrm{h}$, porque el automóvil conducía a $50 \mathrm{~km} / \mathrm{h}$, y puede ver que las dos velocidades eran muy similares. Eso explica por qué se produjo el accidente [seńala los dos vectores a la vez, cuyo módulo es parecido].

Juez: Reproduzcamos el vídeo nuevamente [para ver si los dos vectores son iguales].

En esta discusión, se puede ver que para discutir qué pasó «de verdad» (RA) se usa la información de VA, cuando esta información ha sido directamente definida por la parte que pretende demostrar su versión de los hechos con la simulación.

\section{Nivel de argumentación 2: $S i V D=R D \rightarrow$ demuestro $R A=V A$}

Otros estudiantes optaban por usar la simulación no como una evidencia en sí misma, como en el caso del nivel 1, sino como una evidencia en el caso que el resultado de la colisión virtual (VD), es decir, la posición y velocidad de los vehículos, coincidiera con los datos a los que se enfrentaban (RD). Es decir, usaron la similitud entre los resultados reales (de la ficha) y los virtuales (de la simulación) para justificar así su relato, pero sin contemplar la opción de otros relatos posibles. El objetivo de usar la simulación era, por tanto, validar un único relato.

Este es un extracto del relato de un estudiante que ejerce de acusación:

Acusación: Como podemos ver en esta simulación, el automóvil rojo corresponde a nuestra clienta, y el automóvil verde es el otro conductor. Podemos verificar que la versión de nuestra clienta era verdadera, porque su auto en la simulación termina en el mismo lugar donde ocurrió [en la realidad, según la ficha de su caso]. Podemos observar claramente que la responsabilidad del choque corresponde al auto verde, porque cambió de carril sin atención.

Es decir, como en la simulación los dos coches terminan en el mismo sitio que en la ficha del caso, esto lleva a que su versión sobre qué paso antes de la colisión sea la única versión posible.

\section{Nivel de argumentación 3: Si $V D=R D \rightarrow$ quizás $V A=R A$, pero también puede $V A^{\prime}=R A^{\prime}$}

Finalmente, pudimos observar situaciones en la discusión entre las partes del juicio en las que se confrontaba más de un relato posible de la posición, la velocidad o la trayectoria de los vehículos implicados en el choque (RA, RA'...), todos ellos compatibles con una misma situación final (RD). La siguiente transcripción ejemplifica este tipo de discusiones, en la que una chica que hace de abogada de la defensa intenta demostrar que el coche verde fue arrollado por su cliente debido a que este vehículo iba más deprisa de lo normal, cosa que exculparía a su cliente. En cambio, la acusación usa otra simulación con Collision Lab donde el coche verde iba lento y, aun así, el conductor acusado lo arrolla:

Acusación: No podemos demostrar si su teoría [según la defensa, el coche verde iba demasiado rápido al incorporarse] es verdadera o falsa.

Defensa: ¡Mi cliente me lo dijo!

Juez: ¿Y cómo sabemos que su cliente dice la verdad?

Defensa: ¿Y cómo sabemos que ella [la acusación] dice la verdad?

(Ruido y risas)

Juez: [Al público] ¡Orden! [A la defensa] ¿Qué pruebas tienes? 
Defensa: Con el Collision Lab realizamos una simulación del choque, introdujimos el peso correspondiente y la dirección de cada vehículo, definimos una velocidad más alta para este [vehículo verde], y los resultados coinciden con la posición final de los vehículos en las fotos.

Acusación: ¡Protesto, señoría! ¡Su vídeo termina casi igual que el nuestro! [una grabación de Collision Lab mostrada minutos antes donde el vehículo verde iba mucho más despacio y era arrollado igualmente].

Es decir, a diferencia de lo que ocurre en el nivel de argumentación 2, la coincidencia entre VD de la simulación y RD de la ficha del caso no implica demostrar una versión de RA, sino que puede ser compatible con varias versiones de RA, y por lo tanto solo se podrá usar la simulación como una prueba, si esta apoya una única versión del accidente, no varias.

A diferencia del análisis para la primera pregunta de investigación, para esta segunda cuestión no se ha podido realizar un recuento estadístico del uso de niveles, ya que el discurso de los estudiantes fue cambiando a lo largo de cada discusión, y porque al intervenir varios estudiantes a la vez en algunos casos los patrones se solapaban y evolucionaban de uno a otro. No obstante, como tendencia general, se observó una mayor frecuencia del uso de argumentos de nivel 2, ya que los estudiantes buscaban usar la simulación para que, si era coherente con los datos (RD), automáticamente demostrara su versión de los hechos. Los argumentos de nivel 1 fueron escasos, y a veces eran rebatidos durante el juicio por la otra parte, por los propios jueces (que eran alumnos en el caso del INS Pau Vila, y el profesor en el caso del INS Pius Font i Quer). Finalmente, los argumentos de nivel 3 fueron muy poco comunes al inicio de las presentaciones, pero a medida que la discusión avanzaba, especialmente en el INS Pau Vila, donde esta representaba la celebración de un juicio oral, su presencia fue creciendo en algunas de las discusiones donde se confrontaban versiones de los hechos contradictorias.

\section{CONCLUSIONES E IMPLICACIONES DIDÁCTICAS}

Este estudio ha permitido conocer, en primer lugar, que la simulación Collision Lab ha sido el recurso más elegido como prueba pericial por los estudiantes en ambos centros educativos, a pesar de que el profesorado de ambos centros en ningún momento condicionó su elección, sino que se fomentó que cada grupo de estudiantes eligiera de forma autónoma cómo querían presentar la reconstrucción de los hechos según su relato. Existen posibles causas específicas para esta prevalencia, asociadas a la interactividad y el interés que suscita este tipo de herramientas digitales (Klahr et al., 2006; Honey y Hilton, 2011; Widiyatmoko, 2018). Pero si bien es cierto que revisiones de la literatura como Rutten et al. (2012) señalan que las simulaciones de promedio suscitan una mayor motivación e interés en el alumnado en comparación con otros modos instruccionales, esta y otras revisiones, como las realizadas por Smetana y Bell (2011), ponen el énfasis en que las simulaciones, para mejorar su efectividad educativa, deben usarse para complementar, y no para suplantar otros formatos (como podría ser, en este caso, la experimentación con coches de juguete reales, la elaboración de animaciones manuales del accidente o la realización de gráficos), y también cuando se ofrece al alumnado andamiaje adecuado para su uso, del que hablamos en los siguientes párrafos. Además, este es un elemento para seguir investigando en futuras implementaciones del proyecto CRASH, ya que la mayoría de los estudios previos se han centrado en el uso de simulaciones desde una lógica de indagación y de modelización, y no como un elemento para la resolución de un caso realista como el que nos incumbe, donde lo importante es justamente cómo se conecta el mundo real con el que investigamos y el mundo de las teorías y modelos (Osborne, 2014). En este sentido, la doble naturaleza de las simulaciones propuesta por López et al. (2017) hace de estos instrumentos un recurso muy rico desde la perspectiva de la ciencia escolar, ya que a veces sirven para representar de forma realista el mundo real (actúan de versión de los fenómenos científicos escolares), otras para representar de forma abstracta las explicaciones que la ciencia hace del 
mundo (actúan de versión de los modelos científicos escolares), y otras veces desempeñan un papel híbrido de conexión entre fenómeno y modelo.

En segundo lugar, el estudio ha permitido observar que, a pesar de elegir la simulación como prueba pericial, esta se concibe de distinta forma por parte del alumnado, y que la calidad de las operaciones argumentativas varía según el alumnado y evoluciona a lo largo de una discusión. Hemos visto casos en los que los estudiantes confunden los parámetros que ellos mismos han definido en la simulación con datos que puedan ser usados como prueba (nivel 1), lo que implica un importante error de razonamiento lógico. Otro tipo de argumento, más completo, considera la relación entre los datos reales y los virtuales para justificar su versión de los hechos, aunque sin contemplar más de una opción (nivel 2), que podríamos definir como una operación epistémica de poca calidad (Jiménez-Aleixandre et al., 2003). Finalmente, hemos hallado argumentos que incorporan la idea de grado de similitud entre lo real y lo virtual para argumentar sobre el grado de probabilidad de que una versión sea cierta (nivel 3), de mayor calidad que los anteriores. Los argumentos basados en la simulación dentro de una misma presentación oral son cambiantes, y hemos observado un efecto positivo de la discusión (tanto entre iguales como con el docente) en la evolución de estos argumentos. Por este motivo, consideramos de gran utilidad las guías y andamiajes para promover la discusión crítica de los resultados, como los que apuntan Domènech-Casal et al. (2018), y consideramos que será necesario en el futuro profundizar más en esta cuestión, analizando con mayor detalle la influencia de este andamiaje en la mejora de la calidad de las operaciones argumentativas del alumnado. Hemos podido ver cómo involucrar a los estudiantes en un juego de rol puede promover su participación en prácticas de argumentación, tal como se apunta en la literatura (Casas-Quiroga y Crujérias-Pérez, 2020), y cómo esto, debidamente aprovechado y con una mayor competencia docente para promoverlo, puede ofrecer una oportunidad para mejorar las habilidades argumentativas del alumnado (Driver et al., 2000).

La pandemia de los últimos tiempos, que se suma a otras importantes crisis ecológicas y climáticas, así como el aumento de todo tipo de movimientos negacionistas y conspirativos que arremeten contra las bases de la comunidad científica, hacen más importante que nunca dotar al alumnado de una capacidad de comprender y construir argumentos científicos, no solo desde las certezas absolutas que la ciencia nos puede dar, sino también lidiando con la incertidumbre (Feinstein y Waddington, 2020; Erduran, 2020). A través de instrumentos didácticos como la escala de certidumbres (DomènechCasal, 2019b), se podría involucrar al alumnado, no solo en la resolución de un caso como los que se han presentado en este artículo, sino hacerlo partícipe de una discusión más profunda sobre cómo la ciencia muchas veces debe operar con estas incertidumbres, sin llegar a conocer cuál fue "la verdad». Además, el umbral cada vez más confuso e híbrido entre aquello real y aquello virtual (Cook et al., 2020) también nos lleva a seguir preguntando cómo podemos contribuir, desde de la enseñanza de las ciencias (en este caso, de la física), a una mejora de la competencia de los estudiantes para comprender este diálogo entre lo virtual que se simula a través de ordenador y lo que opera en el mundo real. Entender el papel de las simulaciones desde el punto de vista epistémico es clave para la mejora de esta competencia científica.

\section{AGRADECIMIENTOS}

Esta investigación ha sido financiada por el Ministerio de Ciencia, Innovación y Universidades (PGC2018-096581-B-C21) y llevada a cabo dentro del grupo de investigación ACELEC (2017SGR1399). 


\section{REFERENCIAS BIBLIOGRÁFICAS}

Anta, E. Z., de Barrón, I. C. O., Isasti, L. M. Z. y Escudero, I. E. (2019). Juego de rol para el desarrollo del pensamiento crítico en la formación inicial del profesorado. Revista Complutense de Educación, 30(3), 729.

http://dx.doi.org/10.5209/RCED.58884

Blanco-López, Á., España-Ramos, E. y Franco-Mariscal, A. J. (2017). Estrategias didácticas para el desarrollo del pensamiento crítico en el aula de ciencias. Apice, 1(1), 107-115. https://doi.org/10.17979/arec.2017.1.1.2004

Byrne, J., Heavey, C. y Byrne, P. J. (2010). A review of Web-based simulation and supporting tools. Simulation Modelling Practice and Theory, 18(3), 253-276. https://doi.org/10.1016/j.simpat.2009.09.013

Casas-Quiroga, L. y Crujeiras-Pérez, B. (2020). Epistemic operations performed by high school students in an argumentation and decision-making context: Setrocia's alimentary emergency. International Journal of Science Education, 42(16), 2653-2673. https://doi.org/10.1080/09500693.2020.1824300

Cook, J., Mor, Y. y Santos, P. (2020). Three cases of hybridity in learning spaces: Towards a design for a Zone of Possibility. British Journal of Educational Technology, 51(4), 1155-1167. https://doi.org/10.1111/bjet.12945

Couso, D., Jimenez-Liso, M. R., Refojo, C. y Sacristán, J. A. (Coords.) (2020). Enseñando Ciencia con Ciencia. FECYT y Fundacion Lilly. Madrid: Penguin Random House.

Crujeiras, B. y Jiménez Aleixandre, M. P. (2012). Participar en las prácticas científicas: aprender sobre la ciencia diseñando un experimento sobre pastas de dientes. Alambique. Didáctica de las Ciencias Experimentales, 72, 12-19.

De Jong, T., Linn, M. y Zacharias, Z. (2013). Physical and virtual laboratories in science and engineering education. Science, 340(6130), 305-308. https://doi.org/10.1126/science.1230579

Domènech-Casal, J. (2019a). Aprenentatge basat en projectes, treballs pràctics i controvèrsies. 28 propostes i reflexions per a ensenyar Ciències. Barcelona: Rosa Sensat.

Domènech-Casal, J. (2019b). Escalas de certidumbre y balanzas de argumentos: una experiencia de construcción de marcos epistemológicos para el trabajo con Pseudociencias en secundaria. Ápice, 3(2), 37-53.

https://doi.org/10.17979/arec.2019.3.2.4930

Domènech-Casal, J., Gasco, J., Royo, P. y Vilches, S. (2018). Proyecto CRASH: enseñando cinemática y dinámica en el contexto del análisis pericial de accidentes. Revista Eureka sobre Enseñanza y Divulgación de las Ciencias, 15(2), 210301-210317. http://dx.doi.org/10.25267/Rev_Eureka_ensen_divulg_cienc.2018.v15.i2.2103

Driver, R., Newton, P. y Osborne, J. (2000). Establishing the norms of scientific argumentation in classrooms. Science Education, 84(3), 287-312.

https://doi.org/10.1002/(SICI)1098-237X(200005)84:3<287::AID-SCE1>3.0.CO;2-A

Evagorou, M., Korfiatis, K., Nicolaou, C. y Constantinou, C. (2009). An investigation of the potential of interactive simulations for developing system thinking skills in elementary school: A case study with fifth-graders and sixth-graders. International Journal of Science Education, 31(5), 655-674. https://doi.org/10.1080/09500690701749313

Erduran, S. (2020). Science Education in the Era of a Pandemic. Science \& Education, 29(2), 233-235. https://doi.org/10.1007/s11191-020-00122-w 
Feinstein, N. y Waddington, D. (2020) Individual truth judgments or purposeful, collective sensemaking? Rethinking science education's response to the post-truth era. Educational Psychologist, 55(3), 155-166. https://doi.org/10.1080/00461520.2020.1780130

Fernández, J. E., Ávila, A. M. y Milanés, R. (2017). La Educación vial asistida por tecnología 3D: un modelo de su enseñanza-aprendizaje. Revista Universidad y Sociedad, 9(3), 130-134.

Gilbert, J. K. (2006). On the nature of «context» in chemical education. International Journal of Science Education, 28(9), 957-976. https://doi.org/10.1080/09500690600702470

Glew, R. H. (2003). The problem with problem-based medical school education: Promises not kept. Biochemistry and Molecular Biology Education, 31, 52-56. https://doi.org/10.1002/bmb.2003.494031010158

González-Sánchez, E. (2017). Contribución del juego de rol al desarrollo de la competencia científica en educación secundaria. Percepciones del alumnado participante en una experiencia de juego de rol y dramatización sobre energías alternativas. Enseñanza de las Ciencias, Número Extra X Congreso, 4769-4774.

Gutiérrez, R. (2004). Epistemological and ontological aspects in Science Teacher Education. Quality Development in Teacher Education and Training, 48.

Gutiérrez, R. y Pintó, R. (2005). Relaciones entre simulaciones y modelos: análisis de simulaciones científicas didácticas. Enseñanza de las Ciencias, Número Extra VII Congreso, 1-5.

Hasni, A., Bousadra, F., Belletête, V., Benabdallah, A., Nicole, M. C. y Dumais, N. (2016). Trends in research on project-based science and technology teaching and learning at K-12 levels: a systematic review. Studies in Science Education, 52(2), 199-231.

https://doi.org/10.1080/03057267.2016.1226573

Herreid C. F. (2003) The death of problem-based learning? Journal of College Science Teaching, 32(6), 364-366.

Honey, M. A. y Hilton, M. (2011). Learning science through computer games and simulations. National Academies Press.

Izquierdo, M., Espinet, M., García, M. P., Pujol, R. M. y Sanmartí, N. (1999). Caracterización y fundamentación de la ciencia escolar. Enseñanza de las Ciencias, 17(1), 45-59.

Jiménez-Aleixandre, M. P. y Erduran, S. (2007). Argumentation in science education: An overview. En Argumentation in Science Education (pp. 3-27). Dordrecht: Springer.

Jiménez-Aleixandre, M. P., Reigosa-Castro, C. C. y De Díaz, B. J. (2003). Discourse in the laboratory: quality in argumentative and epistemic operations. In Science Education Research in the Knowledge-Based Society (pp. 249-257). Dordrecht: Springer.

Justi, R. (2006). La enseñanza de ciencias basada en la elaboración de modelos. Enseñanza de las Ciencias, 24(2), 173-184.

Klahr, D., Triona, L. M. y Williams, C. (2007). Hands on what? The relative effectiveness of physical versus virtual materials in an engineering design project by middle school children. Journal of Research in Science Teaching, 44(1), 183-203.

https://doi.org/10.1002/tea.20152

López, V., Couso, D. y Hernández, M. I. (2020). Nuevas miradas sobre el currículo de Física. Alambique, 100, 16-22.

López, V., Couso, D., Simarro, C., Garrido, A., Grimalt, C., Hernández, M. I. y Pintó, R. (2017). El papel de las TIC en la enseñanza de las ciencias en secundaria desde la perspectiva de la práctica científica. Enseñanza de las Ciencias, Número Extra X Congreso, 691-698. 
López, V. y Pinto, R. (2017). Identifying secondary-school students' difficulties when reading visual representations displayed in physics simulations. International Journal of Science Education, 39(10), 1353-1380. https://doi.org/10.1080/09500693.2017.1332441

Matas, A. (2003). Los juegos de rol como recurso formativo. Una aplicación en educación ambiental. Bordón. Revista de Pedagogía, 55(2), 281-291. https://doi.org/10.25267/Rev_educ_ambient_sostenibilidad.2020.v2.i1.1302

Minner, D. D., Levy, A. J. y Century, J. (2010). Inquiry-based science instruction-what is it and does it matter? Results from a research synthesis years 1984 to 2002. Journal of Research in Science Teaching, 47(4), 474-496. https://doi.org/10.1002/tea.20347

Montané, J., Jariot, M. y Rodríguez, M. (2007). Actitudes, cambio de actitudes y conducción segura. Un enfoque crítico aplicado a la reducción de los accidentes de tráfico. Barcelona: Laertes

Olympiou, G. y Zacharias Z. (2012) Blending physical and virtual manipulatives: An effort to improve students' conceptual understanding through science laboratory experimentation. Science Education, 96(1), 21-47. https://doi.org/10.1002/tea.20347

Osborne, J. (2014). Teaching scientific practices: Meeting the challenge of change. Journal of Science Teacher Education, 25(2), 177-196. https://doi.org/10.1007/s10972-014-9384-1

Pérez, M., Couso, D. y Márquez, C. (2020). ¿Cómo diseñar un buen proyecto STEM? Identificación de tensiones en la co-construcción de una rúbrica para su mejora. Revista Eureka sobre Enseñanza y Divulgación de las Ciencias, 18(1), 1301.

https://doi.org/10.25267/Rev_Eureka_ensen_divulg_cienc.2021.v18.i1.1301

Pessanha, M. C. R. (2014). Estrutura da matéria na educação secundária: obstáculos de aprendizagem e o uso de simulaçóes computacionais (tesis doctoral). Universidade de São Paulo (Brasil).

PhET (2020). Collision Lab. https://phet.colorado.edu/es/simulation/collision-lab

Plass, J. L., Milne, C., Homer, B. D., Schwartz, R. N., Hayward, E. O., Jordan, T., Verkullen, J., Ng, F., Wang, Y. y Barrientos, J. (2012). Investigating the effectiveness of computer simulations for chemistry learning. Journal of Research in Science Teaching, 49(3), 394-419. https://doi.org/10.1002/tea.21008

Pons García, L. y de Soto García, I. S. (2020). Evaluación de una propuesta de aprendizaje basado en juegos de rol llevada a cabo en la asignatura de Cultura Científica de Bachillerato. Revista de Estudios y Experiencias en Educación, 19(39), 123-144. http://dx.doi.org/10.21703/rexe.20201939pons7

Rutten, N., van Joolingen, W. y van der Veen, J. (2012) The learning effects of computer simulations in science education. Computers \& Education, 58(1), 136-153. https://doi.org/10.1016/j.compedu.2011.07.017

Ryoo, K. y Linn, M. C. (2012). Can dynamic visualizations improve middle school students' understanding of energy in photosynthesis? Journal of Research in Science Teaching, 49(2), 218-243. https://doi.org/10.1002/tea.21003

Sanmartí, N. y Márquez, C. (2017). Aprendizaje de las ciencias basado en proyectos: del contexto a la acción. Apice, 1(1), 3-16. https://doi.org/10.17979/arec.2017.1.1.2020

Simonneaux, L. (2001). Role-play or debate to promote students' argumentation and justification on an issue in animal transgenesis. International Journal of Science Education, 23(9), 903-927. https://doi.org/10.1080/09500690010016076 
Smetana, L. K. y Bell, R. L. (2012). Computer simulations to support science instruction and learning: A critical review of the literature. International Journal of Science Education, 34(9), 1337-1370. https://doi.org/10.1080/09500693.2011.605182

Vallverdú, J. (2014). What are simulations? An epistemological approach. Procedia Technology, 13, 6-15.

https://doi.org/10.1016/j.protcy.2014.02.003

Widiyatmoko, A. (2018). The Effectiveness of Simulation in Science Learning on Conceptual Understanding: A Literature Review. Journal of International Development and Cooperation, 24(1) 35-43.

Wieman, C., Adams, W. y Perkins, K. (2008). PhET: simulations that enhance learning. Science, 322(5902), 682-683.

https://doi.org/10.1126/science. 1161948

Zacharia, Z. (2007). Combining real and virtual laboratory experimentation: An effort to enhance students' conceptual understanding of electric circuits. Journal of Computer Assisted Learning, 23, 120-132. https://doi.org/10.1111/j.1365-2729.2006.00215.x

Zacharia, Z., Olympou, G. y Papaevripidou, M. (2008). Effects of Experimenting with Physical and Virtual Manipulatives on Students' Conceptual Understanding in Heat and Temperature. Journal of Research in Science Teaching, 45, 1021-1035.

https://doi.org/10.1002/tea.20260 


\title{
Analysis of the use of a collision simulator to solve a traffic accident
}

\author{
Víctor López-Simó \\ Departament de Didàctica de la Matemàtica i les Ciències Experimentals (UAB) \\ David Ferrer Sànchez \\ INS Pius Font i Quer
}

The CRASH project is a PBL in which students are engaged in a case study about traffic accidents. Students receive some information about what happened in a realistic accident, including data such as the final position of the vehicles, the tire tracks, or the declaration of the involved drivers and other witness. With all this information, students must carry out their own inquiries to decide who were the responsible of the crash. At the end of the project students must solve their case in an oral exposition simulating a trial.

In this context, we analyse how these students use a collision simulator called Collision Lab, provided by the PhET Colorado simulation platform. The research questions are: (a) How much is Collision Lab used as an evidence to argue about traffic accidents, in comparison with other types of evidence offered to students?; and (b) which kind of arguments do students make when using the Collision Lab simulation to support their interpretation of the crash?

The results show that Collision Lab is the most used resource by students in their arguments, compared to other resources such as real experiments with toy cars, and much more used than other kinematic analyses such as graphs or calculations made based on the data given to students. However, we have observed that the arguments given by students when using the simulation are often incomplete, and they include misconceptions about the virtual and idealized nature of simulations. Some students use the settings of the simulation (for example, the initial velocity given to the objects to be collided) as if they were real data to justify their point of view. In parallel, some students confuse the fact of «being supportive with a hypothesis» and «demonstrate a hypothesis». Therefore, we discuss the need to address not only the conceptual and procedural understanding of simulations, but also its epistemology. 